\title{
Innovaciones tecnológicas aplicadas al transporte colectivo en Quito. Optimización en la evaluación de la demanda con GPS y SIG
}

Innovations technologiques appliquées au transport en commun à Quito. Optimisation de l'évaluation de la demande à l'aide d'un GPS et d'un SIG Technological innovations applied to the urban transportation in quito. Optimization of evaluation of the demand with gps and gis

Florent Demoraes, Francis Bondoux, Marc Souris y Hidalgo Núñez

\section{(2) OpenEdition}

1 Journals

Edición electrónica

URL: http://journals.openedition.org/bifea/5857

DOI: $10.4000 /$ bifea.5857

ISSN: 2076-5827

Editor

Institut Français d'Études Andines

Edición impresa

Fecha de publicación: 1 abril 2004

Paginación: 193-212

ISSN: 0303-7495

\section{Referencia electrónica}

Florent Demoraes, Francis Bondoux, Marc Souris y Hidalgo Núñez, «Innovaciones tecnológicas aplicadas al transporte colectivo en Quito. Optimización en la evaluación de la demanda con GPS y sIG », Bulletin de l'Institut français d'études andines [En línea], 33 (1) | 2004, Publicado el 08 abril 2004, consultado el 03 diciembre 2020. URL : http://journals.openedition.org/bifea/5857 ; DOI : https:// doi.org/10.4000/bifea.5857

\section{(c)}

Les contenus du Bulletin de l'Institut français d'études andines sont mis à disposition selon les termes de la licence Creative Commons Attribution - Pas d'Utilisation Commerciale - Pas de Modification 4.0 International. 


\title{
INNOVACIONES TECNOLÓGICAS APLICADAS AL TRANSPORTE COLECTIVO EN QUITO
}

\author{
OPTIMIZACIÓN EN LA EVALUACIÓN DE LA DEMANDA CON GPS Y SIG
}

\author{
Florent DEMORAES*, Francis BONDOUX ${ }^{* *}$, Marc SOURIS $^{* * *}$, \\ Hidalgo NÚÑEZ*****
}

\begin{abstract}
Resumen
El presente artículo tiene un triple objetivo. Primero, describir FINDEM, un sistema instalado en las unidades del transporte colectivo provisto con un GPS que permite el conteo semiautomático de pasajeros. Segundo, presentar la metodología de procesamiento de los datos recopilados mediante el uso del Sistema de Información Geográfico SAVANE. Tercero, detallar los principales resultados obtenidos en una primera encuesta Ascensos Descensos realizada con FINDEM en los buses del corredor interparroquial Quito - El Quinche.
\end{abstract}

Palabras claves: Innovación tecnológica, transporte urbano, GPS, SIG, FINDEM, SAVANE, encuesta AD, estudio de demanda, fiscalización, planificación, Quito, Ecuador.

\section{INNOVATIONS TECHNOLOGIQUES APPLIQUÉES AU TRANSPORT EN COMMUN À QUITO}

OPTIMISATION de L'Évaluation DE LA DEMANDE À L'Aide D'Un GPS ET D’Un SIG

\section{Résumé}

Cet article présente un triple intérêt. En premier lieu, il décrit FINDEM, un système embarqué intégrant un GPS permettant le comptage semi-automatique des usagers du transport en commun élaboré à Quito. En second lieu, il présente la méthodologie de traitement des données effectuée avec le Système d'Information Géographique SAVANE. Enfin, sont détaillés

\footnotetext{
" Laboratoire de Géographie - EDYTEM - Université de Savoie - 73376 Le Bouget-duLac Cedex - France. E-mail: fdemo@univ-savoie.fr

** IRD - Université Paul Sabatier - LMTG - 38 rue des 36 Ponts, 31400 Toulouse - France. E-mail: nakamal@wanadoo.com

**** IRD, Institute of Science and Technology for Research and Development, Mahidol University, Nakhon Pathom Province - Thaïlande. E-mail: souris@ird.fr

**** Dirección Metropolitana de Transporte y Vialidad - Municipio del Distrito Metropolitano de Quito, Calle García Moreno 1130 y Chile, Quito - Ecuador. E-mail: hnunez@quito.gov.ec
} 
les principaux résultats obtenus suite à une première enquête " Montées-Descentes " réalisée avec FINDEM dans les bus, le long d'un axe périurbain Quito - El Quinche.

Mots clés Innovation technologique, transport urbain, GPS, SIG, FINDEM, SAVANE, enquête $M D$, étude de demande, contrôle de l'exploitation, planification, Quito, Équateur.

\title{
TECHNOLOGICAL INNOVATIONS APPLIED TO THE URBAN TRANSPORTATION IN QUITO
}

\section{OPTIMIZATION OF EVALUATION OF THE DEMAND WITH GPS AND GIS}

\begin{abstract}
This paper serves three purposes. First, to detail FINDEM, an on-board system developed in Quito, which integrates a GPS and allows a semi-automatic passengers' counting within an urban transportation network. Second, to present the methodology about the processing of the data collected with this equipment using a Geographical Information System called SAVANE. Third, to give the main results of a first "Getting On-Off" survey carried out with FINDEM on buses, along the "Quito - El Quinche" suburban corridor.
\end{abstract}

Key words: Technological innovation, urban transportation, GPS, GIS, FINDEM, SAVANE, "On-Off" survey, demand study, operation control, planning, Quito, Equator.

\section{INTRODUCCIÓN}

Entre los mayores problemas en el ámbito de la transportación urbana que deben enfrentar las municipalidades de muchas metrópolis de países en desarrollo, se encuentran la debilidad de herramienta de control de operación y la escasez de datos básicos referentes a la demanda de transporte por sector. Esas carencias dificultan en particular la planificación y la fiscalización eficaz del transporte colectivo que moviliza cada día a más del $70 \%$ de la población urbana en la mayoría de las grandes ciudades de Latinoamérica, excepto Brasil. En un contexto casi generalizado en Latinoamérica se ha concretado la municipalización de la competencia en materia de transporte urbano y de privatización de la operación (1). Los municipios, entidades concedentes, necesitan disponer de medios técnicos modernos para evaluar la demanda de transporte colectivo y para fiscalizar la operación. Sin esos medios, el transporte puede volverse caótico, como fue el caso en Chile en la década de los 80's con la desregulación total del sector (ver por ejemplo Dourthe et al., 1998). El papel de los cabildos en este sentido es fundamental para garantizar un servicio ciudadano equitativo, seguro, regular y adaptado a la demanda.

En el caso de Quito, “desde finales de 1995, el Municipio del Distrito Metropolitano de Quito tiene toda autoridad en materia de transporte urbano en su

(1) Sin embargo, en la mayoría de las ciudades, en los últimos años la delegación al sector privado fue mínima ya que la participación municipal en el mercado de la operación del transporte colectivo era reducida (Cusset, 1993). 
territorio" (2). El MDMQ se convierte en la autoridad única del transporte, con competencias para "planificar, regular y coordinar todo lo relacionado con el transporte público y privado dentro de su jurisdicción". Dos entes municipales están a cargo de la gestión y administración del transporte: la Dirección Metropolitana de Transporte y Vialidad (DMT) y la Empresa Metropolitana de Servicios y Administración del Transporte (EMSAT). Las principales obligaciones de la DMT, ente rector, son las de "proponer las políticas generales, la planificación y la regulación", "elaborar y actualizar el Plan Maestro de Transporte y Vialidad (PMT)", "proponer el marco regulatorio y la política tarifaria" (3). Las principales obligaciones de la EMSAT, organismo ejecutor, son las de "gestionar, administrar, ejecutar y fiscalizar el tráfico y el transporte en concordancia con el PMT preparado por la DMT, operar, desarrollar y mantener el sistema de señalización y semaforización" (4). Le compete a la EMSAT entregar los permisos de operación a las empresas de transporte.

El Municipio de Quito intenta desde hace varios años desvincularse de la operación (5), fortalecer su papel de planificador, administrador y fiscalizador e incentivar la tercerización de las prestaciones de servicio de transporte. Sin embargo, en el año 2002, sigue operando en forma casi directa, aunque provisionalmente, el trolebús (76 unidades) y la flota de 25 buses de la EMT (Empresa Metropolitana de Transporte). En la región metropolitana de Quito, con una población de 1841000 habitantes (6), se realizan cerca de 2 millones de viajes cotidianamente en transporte colectivo urbano e interparroquial suburbano. Esos viajes representan cerca del $80 \%$ del total de los desplazamientos motorizados diarios lo que muestra la gran importancia del transporte colectivo en el DMQ. La flota de buses del sector privado representa el $97 \%$ del total de unidades operando y participa en el $90 \%$ en la demanda diaria atendida por el transporte colectivo.

El servicio de transporte es relativamente caótico, pues no se respetan las pocas paradas que existen. Tampoco el municipio tiene planos precisos y actualizados de todas las rutas, las cuales cambian de manera recurrente. Una iniciativa común entre el IRD, la DMT, la DMTV y la EMSAT permitió en 2002 elaborar un mapa sinóptico de las rutas urbanas actuales pero falta todavía los itinerarios en las parroquias suburbanas. En total, son cerca de 200 líneas (142 urbanas y 52 interparroquiales).

Como en muchas ciudades, la oferta de transporte público está totalmente atomizada en Quito. El Plan Maestro de Transporte menciona la existencia de 55 operadoras urbanas y 29 interparroquiales que circulan en las mismas vías y entre las cuales existe alta competición que implica carreras entres los buses, contaminación y poca seguridad para los usuarios. El Municipio incentiva la fusión de las cooperativas

(2) El proceso de transferencia de responsabilidades se ha efectuado progresivamente desde que la Ley de Régimen Municipal del Distrito Metropolitano, dictada a finales de 1993, confiere a los poderes municipales de Quito la competencia exclusiva en cuanto a transporte.

(3) Resolución de Alcaldía Nº 002, sancionada el 02 de enero de 2002.

(4) Ordenanza Metropolitana $\mathrm{N}^{\circ}$ 055, sancionada el 13 de junio de 2001.

(5) Esa tendencia se aceleró desde que fue dictada en 1998 la Ley de Modernización del Estado, Privatizaciones y Prestación de Servicios Públicos por parte de la Iniciativa Privada.

(6) Datos según el VI Censo de Población y V de Vivienda (INEC, noviembre de 2001). 
y compañías cuyo funcionamiento es para muchas todavía totalmente artesanal, para crear grandes empresas capitalizadas susceptibles de brindar el servico en un amplio corredor metropolitano. El desafío para el cabildo es poder entregar el servicio a esas empresas privadas disponiendo de datos confiables y actualizados en cuanto a demanda para poder calibrar la oferta necesaria, las frecuencias horarias y los recorridos. Adicionalmente, el municipio está buscando medios técnicos modernos para la fiscalización del servicio que permitirán comprobar si las empresas cumplen con los permisos de operación. El Municipio tiene además la obligación de determinar la tarifas pero le falta datos estadísticos sobre las condiciones operacionales, ingresos y egresos de los operadores.

¿En qué medida el sistema FINDEM puede contribuir a solucionar parte de estos desafíos? ¿Qué tipo de datos permite recolectar? ¿Cúal es el interés de procesarlos en un SIG?

El presente artículo se estructura en tres secciones. Primero se presenta el funcionamiento del sistema FINDEM y sus potencialidades. Segundo se trata de la metodología desarrollada para procesar en el SIG SAVANE los datos de la primera encuesta Ascensos Descensos realizada en Quito con el equipo FINDEM. Finalmente se presenta los principales resultados y conclusiones relevantes del estudio. En el presente documento el término "demanda" se utiliza para referir al uso de la red de transporte colectivo, es decir a la cantidad de personas que utiliza el servicio de transporte.

\section{EL FUNCIONAMIENTO DEL SISTEMA FINDEM Y SUS POTENCIALIDADES}

\section{1. El nacimiento del sistema}

El sistema FINDEM fue inicialmente desarrollado (7) para controlar el recorrido, los tiempos de viajes, los tiempos de parada, las velocidades, el consumo de combustible del transporte de carga en Ecuador. Frente al interés municipal en utilizar FINDEM para evaluar la demanda de transporte, el sistema fue modificado, luego de una serie de reuniones con los gestionarios municipales encargados del transporte colectivo en Quito. Después de las adaptaciones, el sistema fue finalmente utilizado en la ciudad de Quito durante una encuesta ascensos descensos realizada en julio de 2002 en las unidades de transporte interparroquial en el corredor "Quito - El Quinche".

\section{2. Componentes y funcionamiento}

El sistema FINDEM, instalado a bordo del vehículo, funciona en base a los siguientes componentes (8) (véase Fig. 1):

(7) Francis Bondoux ingeniero en sistema del IRD es el diseñador del sistema FINDEM. Siendo un prototipo no existe todavía documentación al respecto. Para mayor información, contáctese con el diseñador. Este instrumento fue inspirado por los trabajos del INRETS (véase referencias citadas).

(8) Se describe aquí la configuración en la que se encontraba FINDEM para la encuesta AD en julio de 2002. Sin embargo el sistema es modificable. Por ejemplo se puede conectar una antena 


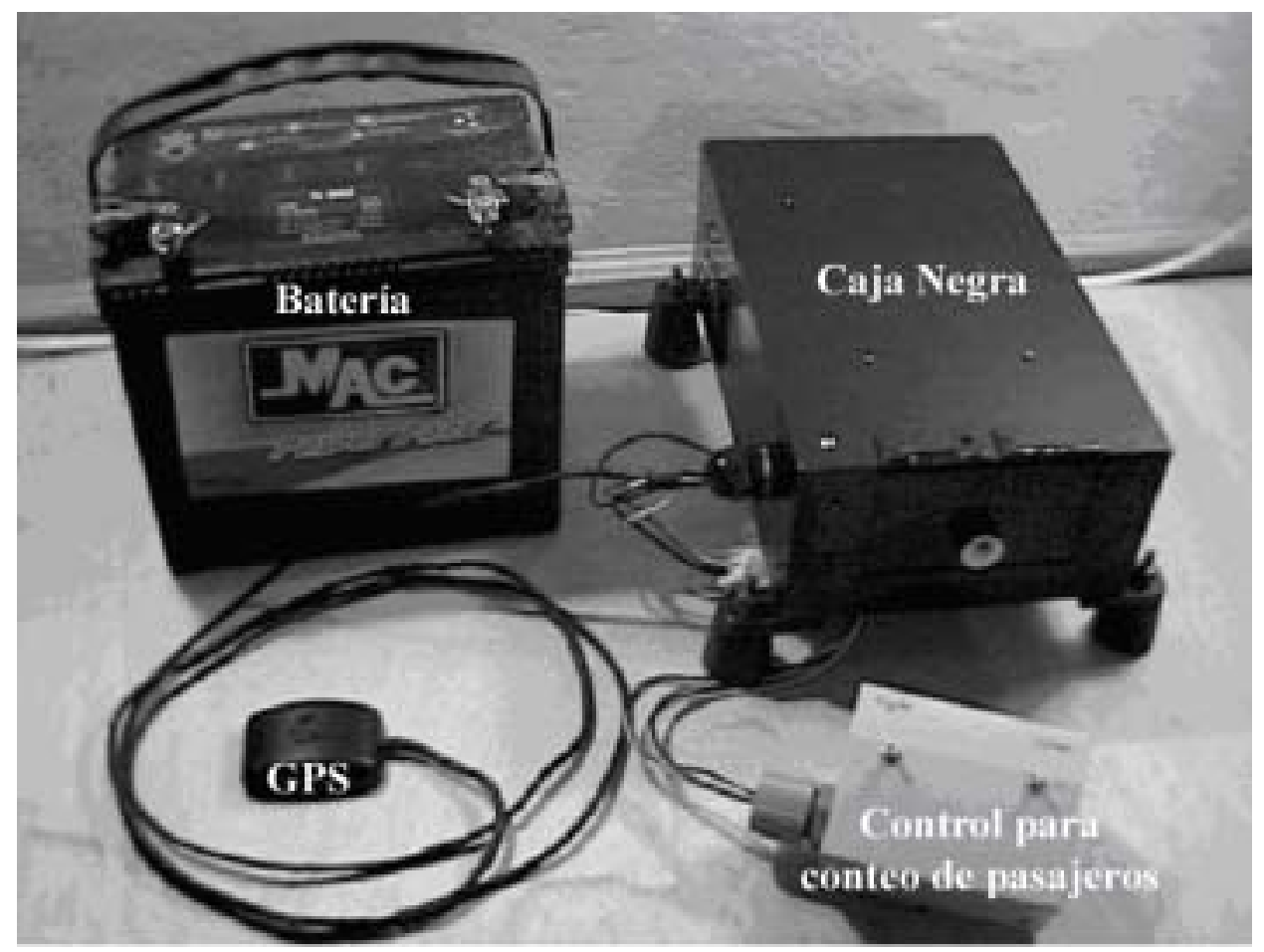

Fig. 1 - Componentes del Sistema FINDEM.

- Un GPS (Global Positioning System) que recibe la señal de los satélites (coordenadas geográficas, $\mathrm{x}, \mathrm{y}, \mathrm{z}$ )

- Un sensor o "control" conectado a la caja negra para que el encuestador instalado a bordo pueda realizar el conteo de los ascensos y descensos de pasajeros

- Una batería externa

- Una tarjeta de adquisición de datos

- Una memoria interna amovible [tarjeta Flash (9)]

- Una batería interna

Nota : esos 3 últimos componentes se ubican en la caja negra "FINDEM".

El GPS se coloca sobre la cubierta del bus y los otros tres componentes en el interior. El sistema está diseñado para realizar un registro cada segundo. Se escogió

que permite enviar directamente los datos registrados a una central de recepción. Esa información puede ser visualizada en tiempo real en un monitor mediante el paquete informático SavFindem desarrollado por Marc Souris. Ensayos preliminares satisfactorios fueron realizados en la ciudad de Quito.

(9) Permite descargar los datos en una computadora portátil por ejemplo. 
esa calibración ya que un pasajero puede subir al bus a cualquier instante. El instrumento recopila dos tipos de datos, unos que son totalmente automáticos y otros que son semiautomáticos, que requieren la intervención del encuestador. Los datos automáticos (formato Tabla Excel) que se recopilan son los siguientes (ver Cuadro1):

- Hora

- Validez del registro (10)

- Posición geográfica (longitud y latitud)

Adicionalmente a partir de los diferentes impulsos realizados por el encuestador a bordo (presión sobre los diferentes botones del sensor) se registran:

- El número de personas que suben y bajan

- Un indicador del sentido del viaje (al iniciar y al concluir un recorrido).

El conteo se realiza con la participación de dos personas instaladas a bordo de la unidad de transporte. Una cuenta y verifica los ascensos y descensos, otra registra los pasajeros con el sensor.

A partir de la recopilación de los datos digitales iniciales se pueden efectuar varios procesamientos con la utilización en especial de un SIG que permita dibujar planos de los recorridos de los buses y calcular:

- La distancia de un viaje

- Su duración

- La velocidad instantánea y promedia del vehículo

- Los lugares de mayor afluencia de pasajeros (tramos de mayores ascensos)

- Las horas pico de mayor demanda

- Los tramos con mayor carga de pasajeros, etc...

El uso de FINDEM es innovador ya que es un sistema semiautomático. Es decir, permite el levantamiento del recorrido y la medición de velocidades de forma íntegramente automática, mientras el conteo es realizado por el encuestador (11). FINDEM facilita de manera innegable la tarea. Permite optimizar la realización de una encuesta AD. Es decir, ya no hace falta las anotaciones manuales en fichas de campo por parte de los encuestadores, las cuales debían posteriormente ser ingresadas en formato digital lo que implicaba sesgos y errores además de ser un procedimiento largo. El sistema FINDEM está adaptado al contexto de ciudades en las cuales no se respetan las pocas paradas de bus, como es el caso de Quito (12). Es decir, con la

(10) Al encender el equipo, se debe esperar unos segundos para recibir la señal satelital. Mientras no sea suficiente el número de satélites reconocidos por el GPS, no es posible iniciar la grabación de los datos; esto permite evitar datos erróneos.

(11) Primero, para hacer encuestas móviles, se necesitaba un equipo liviano de fácil traslado. Luego, era imposible encontrar un equipo que se podía adaptar rápidamente a todos los buses, siendo muy heterogénea la flota. En fin, la operación puede ser caótica y la tasa de ocupación de los vehículos es a veces muy elevada.

(12) El Municipio instaló 1200 paradas en la ciudad en el 2001 y 2002, pero no siempre se las respetan. En el corredor Quito - El Quinche son pocas paradas, pero tampoco se las respetan. 
Cuadro 1 - Ejemplo de datos iniciales grabados por FINDEM el día 1.08.2002 (se indica aquí únicamente las primeras filas del cuadro).

\begin{tabular}{|c|c|c|c|c|c|c|c|c|}
\hline clave & $\begin{array}{l}\text { Lat } D^{o} \\
\text { decim }\end{array}$ & $\begin{array}{c}\text { Long } D^{o} \\
\text { decim }\end{array}$ & Hora & Suben & Bajan & Quedan & $\mathbf{N}^{\mathrm{a}}$ viaje & Sentido \\
\hline 1 & $-0,2011$ & $-78,3754$ & $6: 44: 59$ & 1 & 0,0 & 1 & viaje 1 & Arenal $\longrightarrow$ Quito \\
\hline 2 & $-0,2011$ & $-78,3754$ & $6: 45: 00$ & 1 & 0,0 & 2 & viaje 1 & Arenal $\longrightarrow$ Quito \\
\hline 3 & $-0,2011$ & $-78,3754$ & $6: 45: 01$ & 1 & 0,0 & 3 & viaje 1 & Arenal $\longrightarrow$ Quito \\
\hline 4 & $-0,2011$ & $-78,3754$ & $6: 45: 02$ & 1 & 0,0 & 4 & viaje 1 & Arenal $\longrightarrow$ Quito \\
\hline 5 & $-0,2011$ & $-78,3754$ & $6: 45: 03$ & 1 & 0,0 & 5 & viaje 1 & Arenal $\longrightarrow$ Quito \\
\hline 6 & $-0,2011$ & $-78,3754$ & $6: 45: 04$ & 1 & 0,0 & 6 & viaje 1 & Arenal $\longrightarrow$ Quito \\
\hline 7 & $-0,2011$ & $-78,3754$ & $6: 45: 05$ & 1 & 0,0 & 7 & viaje 1 & Arenal $\rightarrow$ Quito \\
\hline 8 & $-0,2011$ & $-78,3754$ & $6: 45: 06$ & 1 & 0,0 & 8 & viaje 1 & Arenal $\longrightarrow$ Quito \\
\hline 9 & $-0,2011$ & $-78,3755$ & $6: 45: 07$ & 1 & 0,0 & 9 & viaje 1 & Arenal $\longrightarrow$ Quito \\
\hline 10 & $-0,2011$ & $-78,3755$ & 6:45:08 & 1 & 0,0 & 10 & viaje 1 & Arenal $\longrightarrow$ Quito \\
\hline 11 & $-0,2011$ & $-78,3755$ & $6: 45: 09$ & 1 & 0,0 & 11 & viaje 1 & Arenal $\longrightarrow$ Quito \\
\hline 12 & $-0,2011$ & $-78,3755$ & $6: 45: 10$ & 1 & 0,0 & 12 & viaje 1 & Arenal $\longrightarrow$ Quito \\
\hline 13 & $-0,2011$ & $-78,3755$ & $6: 45: 11$ & 1 & 0,0 & 13 & viaje 1 & Arenal $\longrightarrow$ Quito \\
\hline 14 & $-0,2011$ & $-78,3755$ & $6: 45: 12$ & 1 & 0,0 & 14 & viaje 1 & Arenal $\rightarrow$ Quito \\
\hline 15 & $-0,2011$ & $-78,3755$ & $6: 45: 13$ & 1 & 0,0 & 15 & viaje 1 & Arenal $\rightarrow$ Quito \\
\hline 16 & $-0,2011$ & $-78,3755$ & $6: 45: 14$ & 1 & 0,0 & 16 & viaje 1 & Arenal $\longrightarrow$ Quito \\
\hline 17 & $-0,2011$ & $-78,3756$ & $6: 45: 15$ & 1 & 0,0 & 17 & viaje 1 & Arenal $\longrightarrow$ Quito \\
\hline 18 & $-0,2011$ & $-78,3756$ & $6: 45: 16$ & 1 & 0,0 & 18 & viaje 1 & Arenal $\rightarrow$ Quito \\
\hline 19 & $-0,2011$ & $-78,3756$ & $6: 45: 17$ & 0,0 & 0,0 & 18 & viaje 1 & Arenal $\longrightarrow$ Quito \\
\hline 20 & $-0,2011$ & $-78,3756$ & $6: 45: 18$ & 0,0 & 0,0 & 18 & viaje 1 & Arenal $\longrightarrow$ Quito \\
\hline 21 & $-0,2011$ & $-78,3756$ & $6: 45: 19$ & 0,0 & 0,0 & 18 & viaje 1 & Arenal $\longrightarrow$ Quito \\
\hline 22 & $-0,2011$ & $-78,3756$ & $6: 45: 20$ & 0,0 & 0,0 & 18 & viaje 1 & Arenal $\rightarrow$ Quito \\
\hline 23 & $-0,2012$ & $-78,3756$ & $6: 45: 21$ & 0,0 & 0,0 & 18 & viaje 1 & Arenal $\longrightarrow$ Quito \\
\hline 24 & $-0,2012$ & $-78,3756$ & $6: 45: 22$ & 0,0 & 0,0 & 18 & viaje 1 & Arenal $\rightarrow$ Quito \\
\hline 25 & $-0,2012$ & $-78,3755$ & $6: 45: 23$ & 0,0 & 0,0 & 18 & viaje 1 & Arenal $\rightarrow$ Quito \\
\hline 26 & $-0,2012$ & $-78,3755$ & $6: 45: 24$ & 0,0 & 0,0 & 18 & viaje 1 & Arenal $\longrightarrow$ Quito \\
\hline 27 & $-0,2012$ & $-78,3755$ & $6: 45: 25$ & 0,0 & 0,0 & 18 & viaje 1 & Arenal $\longrightarrow$ Quito \\
\hline 28 & $-0,2012$ & $-78,3755$ & $6: 45: 26$ & 0,0 & 0,0 & 18 & viaje 1 & Arenal $\longrightarrow$ Quito \\
\hline 29 & $-0,2012$ & $-78,3755$ & $6: 45: 27$ & 0,0 & 0,0 & 18 & viaje 1 & Arenal $\longrightarrow$ Quito \\
\hline 30 & $-0,2012$ & $-78,3755$ & $6: 45: 28$ & 0,0 & 0,0 & 18 & viaje 1 & Arenal $\rightarrow$ Quito \\
\hline 31 & $-0,2012$ & $-78,3755$ & $6: 45: 29$ & 0,0 & 0,0 & 18 & viaje 1 & Arenal $\longrightarrow$ Quito \\
\hline 32 & $-0,2012$ & $-78,3755$ & $6: 45: 30$ & 0,0 & 0,0 & 18 & viaje 1 & Arenal $\longrightarrow$ Quito \\
\hline 33 & $-0,2012$ & $-78,3755$ & $6: 45: 31$ & 0,0 & 0,0 & 18 & viaje 1 & Arenal $\rightarrow$ Quito \\
\hline 34 & $-0,2012$ & $-78,3755$ & $6: 45: 32$ & 0,0 & 0,0 & 18 & viaje 1 & Arenal $\rightarrow$ Quito \\
\hline 35 & $-0,2012$ & $-78,3755$ & $6: 45: 33$ & 0,0 & 0,0 & 18 & viaje 1 & Arenal $\rightarrow$ Quito \\
\hline 36 & $-0,2012$ & $-78,3755$ & $6: 45: 34$ & 0,0 & 0,0 & 18 & viaje 1 & Arenal $\longrightarrow$ Quito \\
\hline
\end{tabular}


participación, únicamente de dos encuestadores a bordo de las unidades se puede determinar con precisión, dónde, cuándo, y cuántos pasajeros suben y bajan de los buses.

\section{EL USO DE FINDEM EN UNA ENCUESTA ASCENSOS DESCENSOS, PRESENTACIÓN METODOLÓGICA DEL PROCESAMIENTO DE DATOS EN EL SIG SAVANE}

\section{1. Antecedentes de la encuesta}

La serie de encuestas de Ascensos Descensos realizadas (13) en el corredor Quito - El Quinche con el equipo FINDEM corresponde a un estudio puntual de determinación de la demanda en transporte colectivo pedido por la Dirección Metropolitana de Transporte y Vialidad del Municipio de Quito y realizado por Bondoux $\&$ Demoraes (2002). Se inserta en un proceso de evaluación general de la demanda en transporte colectivo en el área metropolitana cuyo objetivo es contribuir a ajustar un modelo de racionalización y modernización del transporte metropolitano. Este modelo tiene como objetivos:

- Planificar y orientar la política del transporte en la aglomeración capitalina

- Calibrar adecuadamente la oferta con la demanda con el fin de delegar la operación a empresas grandes

- Dimensionar algunas infraestructuras tales como las paradas y terminales de transferencia planificadas en el Plan Maestro de Transporte y Vialidad (MDMQDMT, 2002)

- Evaluar los costos de operación y las tarifas del transporte público (14).

Además permite fiscalizar el servicio brindado por las cooperativas y/o compañías de transporte del corredor es decir, comprobar si cumplen con los permisos de operación otorgados por la EMSAT.

\section{1. 1. Cobertura de la encuesta}

La encuesta fue realizada a 13 rutas de transporte colectivo interparroquial (15) (Véase Fig. 2), es decir el $25 \%$ del total de las rutas suburbanas.

(13) Las encuestas se hicieron desde las 6:00 hasta las 19:30 en días laborables a partir del jueves 04/07/2002 hasta el viernes 02/08/2002.

(14) Hasta la fecha, las tarifas vigentes para los trayectos interparroquiales son las fijadas por el Consejo Nacional de Tránsito, sin verdaderos criterios técnicos. Sin embargo, desde 1995, le compete al Municipio de Quito determinar todas las tarifas del transporte colectivo metropolitano. De ahí, la necesidad de disponer de datos estadísticos actualizados sobre los costos operacionales.

(15) Se trata de las líneas que brindan un servicio de transporte hasta las cabeceras parroquiales suburbanas y pueblos siguientes: El Quinche, Cusubamba, La Victoria, Yaruquí, Pifo, Puembo, Mangahuantag, San José, El Arenal, Collaquí, La Morita, La Primavera, La Comuna, Lumbisí, San Juan. 
Red de transporte interparroquial - Quito / El Quinche

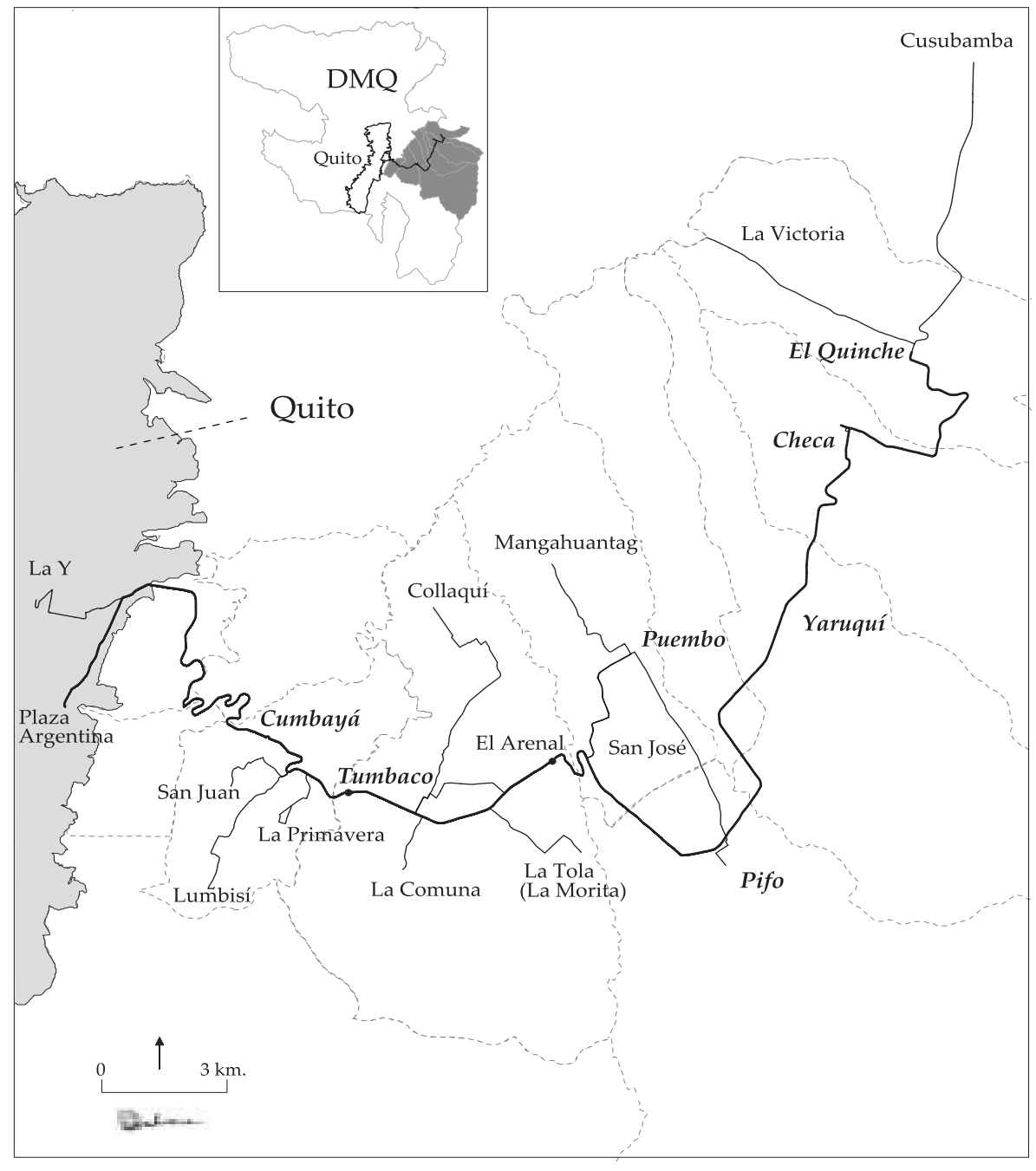

Fig. 2 - Representación cartográfica sinóptica de las rutas del estudio de determinación de la demanda en transporte interparroquial del corredor Quito - Quinche.

Se realizó una encuesta $\mathrm{AD}$ por hora, por sentido y por ruta. La frecuencia máxima de operación observada, es decir el mayor número de buses que salieron de su despacho, fue de 10 unidades por hora por ruta, por lo tanto el muestreo corresponde a un $10 \%$ como mínimo (16).

(16) Según lo estableció la DMT. 
El corredor cubre 8 parroquias suburbanas y cuenta con una población de $120 \quad 000$ personas, es decir el 7\% de la población distrital. Entre el inicio del corredor principal (Plaza de Argentina) y el final (El Quinche) se cuenta una distancia de unos 50 kilómetros.

\section{2. Metodología de procesamiento de datos}

\section{2. 1. Consideraciones preliminares}

La información grabada por FINDEM corresponde al posicionamiento del vehículo con sus diferentes variables tales como los ascensos, descensos... en un instante "t" (ver Cuadro 1). Esa información puede entonces ser integrada a un Sistema de Información Geográfico para que pueda ser espacializada. Sin embargo, esos datos no son directamente utilizables porque son muy numerosos (17). Lo que se busca más bien son las condiciones operacionales a lo largo de los recorridos de bus es decir, por tramo para que sea más comprensible y útil. Una manera de resumir y sintetizar la información de los puntos es la geoagregación o agregación espacial por tramo vial que permite tener una condición promedio de operación. Este tratamiento se lo puede hacer únicamente en un SIG.

A continuación se describen las sucesivas etapas de procesamiento de datos realizado con el Sistema de Información Geográfico SAVANE (18). Este SIG es utilizado por la Dirección Metropolitana de Territorio y Vivienda del Municipio de Quito desde principios de la década de los 90. Este sistema contiene una amplia base de datos (socioeconómicos, demográficos, de infraestructura, entre otros) útil para la gestión y planificación territorial. La Unidad de Estudios e Información Metropolitana en colaboración con el IRD ha mantenido y actualizado la información en el SIG desde el año 2000, dentro del marco del proyecto de investigación "Sistema de Información y Riesgos en el Distrito Metropolitano de Quito". Los mapas viales y los fondos cartográficos utilizados en este estudio provienen de esa geobase.

\section{2. 2. Etapa 1}

Los datos iniciales (ascensos, descensos, posición geográfica, hora, indicadores de sentido...) que se registraron en FINDEM fueron transferidos de la tarjeta Flash a una computadora y depurados en Excel. Luego, estos datos fueron integrados en una nueva "relation" a la base SAVANE (integración de puntos) (19). Una "relation" contiene datos alfanuméricos y cartográficos asociados. Para explicar mejor en función de otros SIGs esto corresponde más o menos a una "Table" en MapInfo o a un "Theme" en ArcView (20).

(17) Cada día de registro genera una base de datos de aproximadamente unas 50000 filas en Excel ( 1 hora $=3600$ segundos o sea 3600 filas de registro multiplicadas por 14 horas diarias de registro). Ver Cuadro 1.

(18) Marc Souris, informático e investigador del IRD, es el diseñador del SIG Savane.

(19) Para mayor información en cuanto a la utilización del SIG Savane, véase la documentación referenciada en la bibliografía.

(20) Sin embargo, el esquema de Savane está estructurado en una forma diferente, de tipo "objeto", es decir sin separación entre los datos alfanuméricos y cartográficos. 


\section{2. 3. Etapa 2}

En el SIG se extrajeron los puntos entre los cuales existía una distancia de 200 metros. Estos puntos fueron unidos es decir, convertidos en segmentos que sirven como referencia (21). Se escogieron tramos de referencia relativamente cortos en el sentido de que las condiciones operacionales fluctúan sustancialmente a lo largo del corredor en cortas distancias. Además se necesitaban tramos codificados con una longitud igual para poder comparar los tramos entre sí. Ello posibilita un análisis muy detallado y una comprensión fina del comportamiento operacional de las diferentes rutas. Esos datos muy desagregados permiten luego hacer agrupaciones por parroquias, por barrio, por cabeceras suburbanas, o por zonas de modelación de transporte...

\section{2. 4. Etapa 3}

La información integrada como "relation" puntual fue geoagregada en esos tramos de referencia como se detalla a continuación.

Se realizaron dos tipos de geoagregaciones con un nivel de precisión de 25 metros. Esa precisión se la puede determinar en Savane, lo que no es el caso en todos los demás SIGs. Se escogió una franja de 25 metros de ambos lados del recorrido para hacer las geoagregaciones porque el posicionamiento de los vehículos no siempre se sobrepone exactamente en los tramos viales por el hecho de que la señal satelital suele distorsionarse levemente (por el número de satélites presentes en el cielo) y también por el hecho de que el mapa vial que sirve de referencia tiene un margen de error (desfases geográficos). Consecuentemente, los valores de los puntos ubicados en las extremidades de los tramos (en los 25 primeros y últimos metros) están contabilizados también en los tramos adyacentes. Es decir, existe una mínima duplicación de datos que no excede el $10 \%$ de los valores reales (22) y que no cambia el comportamiento general de la demanda a lo largo del recorrido y su variación a lo largo del día. Los dos tipos de geoagregaciones son los siguientes:

- para obtener la demanda por tramo se calculó el promedio de la carga de pasajeros que se tiene para cada segundo (promedio del número de pasajeros presentes en el bus en cada tramo);

- para los ascensos y descensos, en cambio se contabilizaron todas la personas que subieron o bajaron en cada tramo de 200 metros (suma).

\section{2. 5. Etapa 4}

Finalmente se procedió a la expansión de la muestra. Se multiplicaron simplemente los datos iniciales obtenidos durante el conteo de un viaje, geoagregados en los tramos, por el número de buses que salieron en la hora correspondiente (datos de un viaje multiplicados por la frecuencia horaria de operación).

(21) Eso nos permitió evitar la digitalización manual de los recorridos.

(22) De hecho en la práctica, se observó que las subidas y las bajadas de los pasajeros no eran muy frecuentes en esas extremidades. 


\section{PRINCIPALES RESULTADOS Y CONCLUSIONES RELEVANTES}

Con el levantamiento de las rutas se pudo primero elaborar un mapa de la red de transporte colectivo en el corredor Quito - El Quinche, lo cual no existía hasta entonces (23).

\section{1. Demanda total del corredor}

El total diario de pasajeros transportados en el corredor en las 13 rutas interparroquiales en el sentido Quito -> El Valle asciende a 55 000. El total diario de pasajeros transportados en el corredor en las 13 rutas interparroquiales en el sentido El Valle -> Quito asciende a 53300.

En total se movilizan diariamente unas 108300 personas en las 13 rutas interparroquiales que operan en el corredor Quito - El Quinche en ambos sentidos. Según los datos preliminares del censo del INEC (2001), 119360 personas viven en las 8 parroquias suburbanas correspondientes al corredor. Significa un índice de movilidad en transporte colectivo de 0,9 viajes por habitante por día para este sector. En cambio, el índice de movilidad en transporte colectivo es de 1,2 (24) en la parte urbana de Quito. El uso del transporte colectivo en el corredor suburbano parece entonces menor que en la ciudad. Sin embargo, sin estudios adicionales sobre la tasa de motorización por hogar, sobre las prácticas de movilidad y el uso del transporte informal (25), es difícil determinar cuál es la proporción entre los diferentes tipos de movilidad.

\section{2. Demanda versus oferta horaria}

\section{2. 1. Horas pico}

Como es el caso en muchas ciudades, las horas pico corresponden a los horarios de funcionamiento de las actividades socioeconómicas (oficinas, escuelas, comercios, servicios, labor agrícola). La mayor demanda se da en la mañana y en la tarde. No se observa un pico secundario de demanda al medio día. Significa que la mayoría de la gente no realiza más de 2 viajes por día, quedándose a comer en el lugar en el que se encuentra al mediodía, sin regresar a su casa.

En la mañana en el sentido Quito -> El Valle, la mayor demanda se da en la hora 8 - 9 en todo el corredor con unas 5108 personas transportadas en las 13 rutas interparroquiales. En la tarde la mayor demanda en el mismo sentido ocurre en la hora 16 - 17 con unas 6178 personas transportadas.

(23) También se pudo determinar las velocidades promedias de operación por tramo. Este tema no se presenta en el presente texto.

(24) La zona urbana de Quito cuenta con una población de 1426190 habitantes (población del distrito menos población de las parroquias rurales, datos preliminares del censo - INEC, 2001) y se registra cada día 1709800 viajes urbanos eћC (total de los viajes metropolitanos menos los viajes interparroquiales y escolares).

(25) 2550 hogares de bajos ingresos (consumo de luz inferior a $120 \mathrm{KW} / \mathrm{h}$ por mes) se encuentran a más de 700 metros de una ruta de transporte interparroquial regular en las 8 parroquias suburbanas del Valle de Cumbayá - El Quinche (valor calculado a partir de los datos de la Empresa Eléctrica Quito). Se puede estimar que el uso del transporte informal es entonces alto. 
En la mañana en el sentido El Valle -> Quito, la hora de mayor demanda es la hora 6 - 7 en todo el corredor con unas 6020 personas transportadas. En la tarde la hora de mayor demanda en el mismo sentido es la hora 17 - 18 con unas 5122 personas transportadas.

Las horas pico no coinciden entre los 2 sentidos. Estudios adicionales tales como encuestas de origen y destino con los motivos de viajes, permitirían entender con mayor precisión las principales dinámicas de la demanda del corredor.

\section{2. 2. Oferta horaria}

La oferta o la frecuencia de operación horaria de los buses es casi constante a lo largo del día (por lo menos hasta las 19:00 horas). En cambio la demanda fluctúa sustancialmente en función de la hora del día (Figs. 3 y 4). Ello pone en evidencia la inadecuación de la oferta en relación con la demanda. Durante gran parte del día, se podría decir que el número de unidades operando es suficiente, e incluso demasiado alto, para asegurar condiciones aceptables de servicio. Las unidades cuya capacidad varía entre 45 y 50 asientos, pueden circular con apenas 15 personas a bordo en las horas de baja demanda lo que representa un desperdicio de energía y una sobreocupación inútil de la vías. En cambio, ya no es el caso en las horas de mayor demanda, en las que las unidades circulan a más del $100 \%$ de su capacidad. Pueden transportar hasta 80 pasajeros de los cuales unos 30 están parados lo que es muy peligroso además de ser prohibido. Ello se observa en la tarde entre las 16 y 19 horas en el sentido Quito -> El Valle y en la mañana entre las 6 y 7 horas en el sentido El Valle -> Quito. La oferta no se adapta a la demanda.

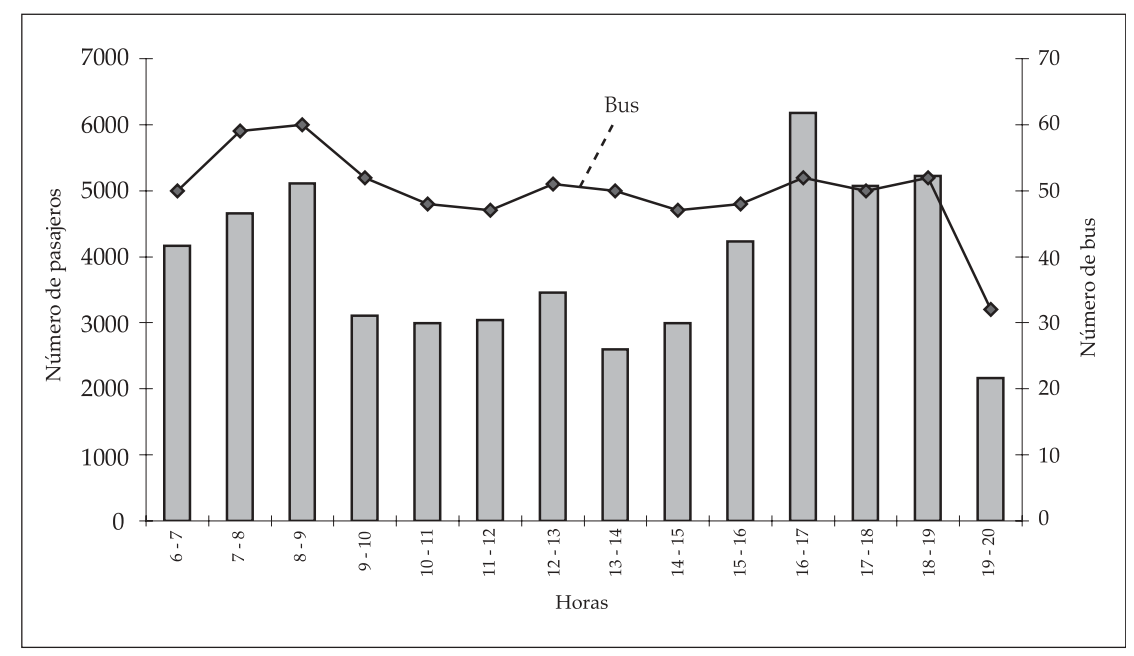

Fig. 3 - Total de pasajeros transportados en el corredor principal y en las extensiones (13 rutas interparroquiales) por hora y frecuencia de operación / Sentido Quito -> El Valle. 


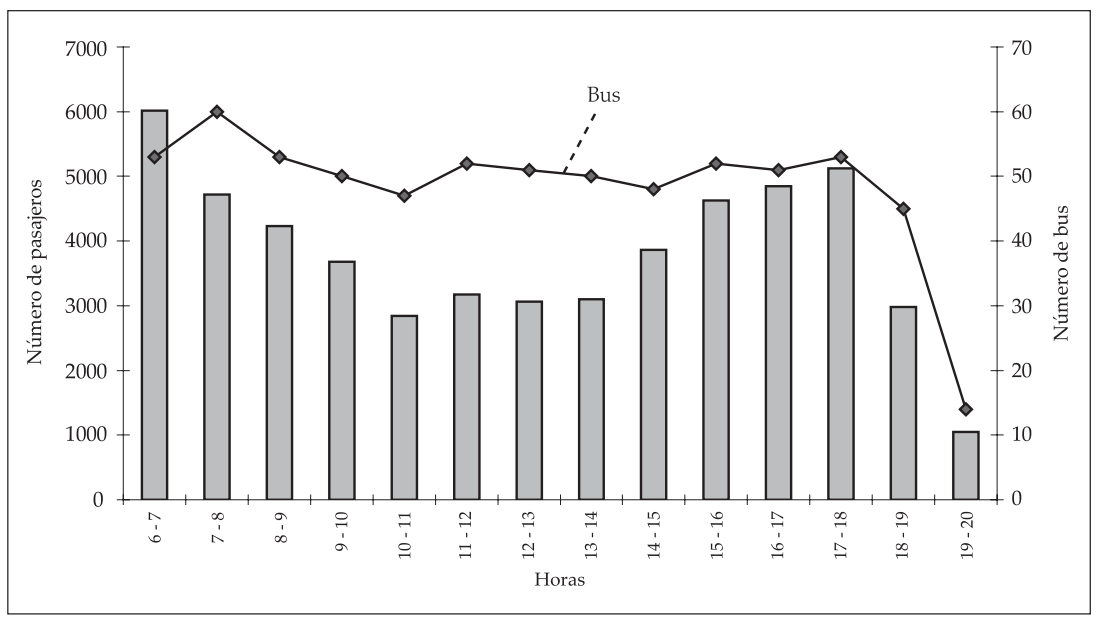

Fig. 4 - Total de pasajeros transportados en el corredor principal y en las extensiones (13 rutas interparroquiales) por hora y frecuencia de operación / Sentido El Valle -> Quito.

\section{3. Demanda y Ascensos Descensos diarios a lo largo del corredor}

11 rutas encuestadas sobre 13 pasan por el corredor principal Quito - El Quinche (26) por lo menos en parte de su recorrido. Para evaluar, en esas secciones comunes, la demanda, los ascensos y descensos totales diarios, se sumaron por tramo los valores diarios que se tiene para cada una de las 11 rutas. Para este cálculo, se utilizó un método de tratamiento en el SIG llamado unión (jointure) (27) en base a las "claves" o códigos de los tramos que sirven como atributo de unión.

Como ejemplo, se presenta un mapa sinóptico (Fig. 5) que muestra la demanda total diaria por tramo de 200 metros en el corredor principal y en las extensiones para el sentido Quito -> El Valle.

La sección de mayor demanda es la que está comprendida entre la salida de Quito (Granados y Eloy Alfaro) y Tumbaco con más de 15000 pasajeros diarios. Estos sectores son los más poblados (28) y cuentan con centros comerciales, servicios, escuelas y una universidad.

La demanda se dispersa progresivamente primero a la salida de Cumbayá hacia los barrios San Juan, Lumbisí y La Primavera (esencialmente residenciales). A partir de Tumbaco existen 4 extensiones hacia los barrios La Comuna, Collaquí, La Morita,

(26) El corredor principal está conformado por la Interoceánica y luego por la Perimetral Metropolitana hacia El Quinche a partir de la Y de Pifo.

(27) $\mathrm{Al}$ constituir los recorridos de cada ruta en el SIG es importante que, en las secciones comunes, los tramos tengan la misma codificación. Además es fundamental que cada objeto tenga un código propio y único.

(28) Tumbaco cuenta con una población de 38550 habitantes y Cumbayá con 21100 (datos preliminares Censo 2001, INEC). 
Demanda total diaria en el corredor principal y en las extensiones por tramo de 200 metros (Cúmulo de las 11 rutas)

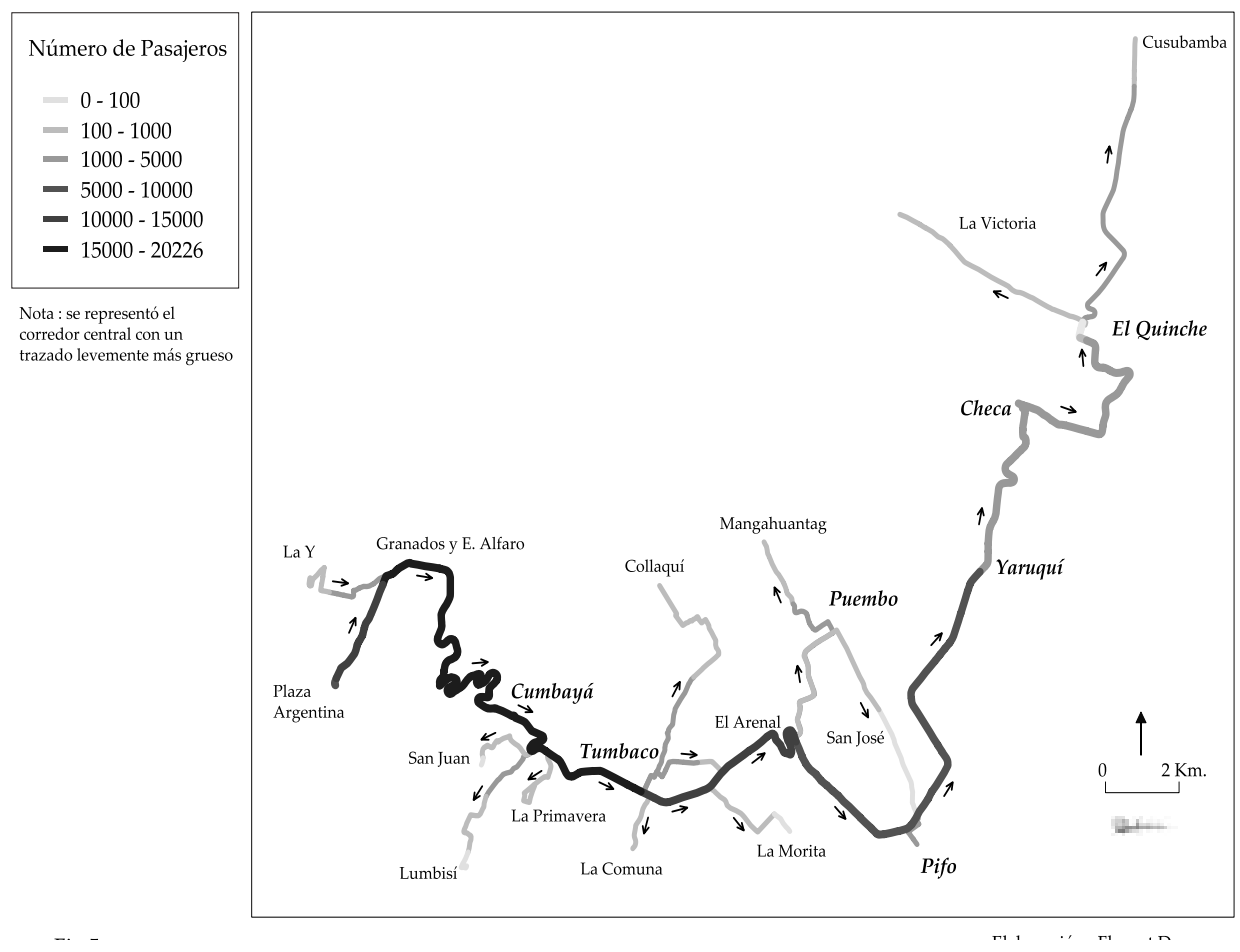

Fig. 5 - Representación de la demanda diaria por tramo a lo largo del corredor Quito -> El Quinche.

El Arenal. En el corredor central la demanda sigue alta hasta Yaruquí (entre 5000 y 10000 pasajeros diarios).

De una manera general, la demanda va decreciendo mientras se aleja del corredor principal salvo en Puembo en donde se observa un incremento de demanda a partir del parque central hacia Mangahuantag (viajes locales).

La demanda diaria en las extensiones no sobrepasa $2 \quad 860$ pasajeros (caso de la extensión entre la Y de Puembo y el parque central de Puembo).

Los ascensos y descensos por tramo presentados en el figura 6 corresponden únicamente a los que son realizados en el corredor principal. Ello implica que parte se realizan fuera del corredor principal por lo cual la suma de los ascensos y descensos totales diarios no coincide.

Los principales lugares en los que se observa la mayor afluencia de pasajeros son en orden decreciente (Véase Fig. 6): 


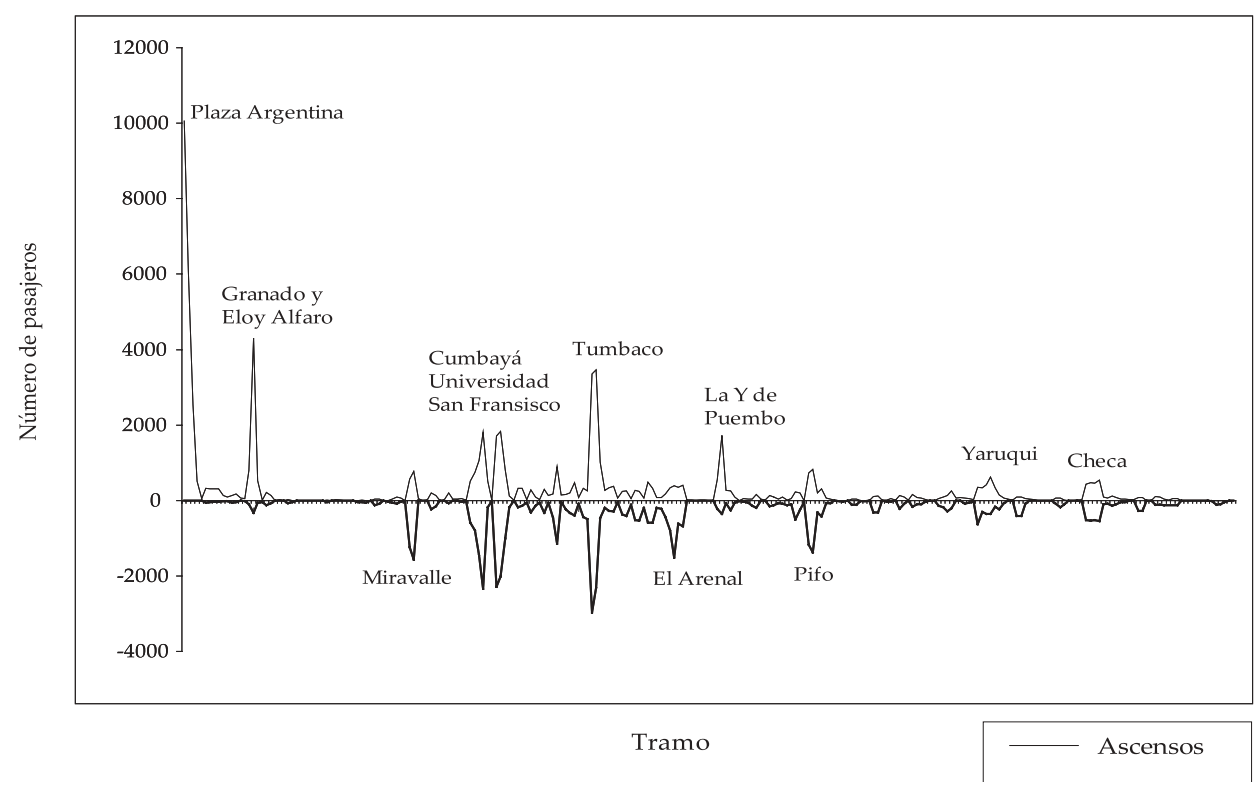

Fig. 6 - Ascensos Descensos diarios totales en el corredor principal por tramo sentido Quito -> El Valle (cúmulo de las 11 rutas). (Nota: parte de los ascensos y descensos se realizan fuera del corredor principal, es decir en las extensiones no representadas aquí.)

- La Plaza de Argentina con más de 10000 ascensos diarios (inicio de las rutas)

- La salida de Quito (Granados y Eloy Alfaro) con más de 4000 ascensos diarios

- El semáforo del parque central de Tumbaco con más de 3300 ascensos diarios

- El semáforo de Cumbayá y la Y de Puembo con más de 1700 ascensos diarios

Los principales lugares en los que se observa los mayores descensos de pasajeros son en orden decreciente (Fig. 6):

- El semáforo del parque central de Tumbaco con cerca de 3000 descensos diarios

- El redondel de la Universidad San Fransisco y el semáforo de Cumbayá con más de 2300 descensos diarios

- El Arenal con 1500 descensos diarios

En el sentido Quito - El Valle, los descensos se realizan de una manera más equitativa que los ascensos a lo largo del corredor (no existe por ejemplo un pico de descensos como el pico de ascensos de la Plaza de Argentina). En la parte central del corredor (Cumbayá, Tumbaco), muchos lugares de gran afluencia coinciden con los de mayores descensos. 


\section{4. Llegadas y salidas diarias a/de Quito}

En la entrada a Quito (redondel de la vía a Nayón) llegan del valle 20156 personas diariamente en las 11 rutas interparroquiales que hacen la conexión Quito - EValle sobre un total diario de 53400 usuarios transportados en el corredor. Significa que más del $62 \%$ de los viajes se realizan al interior del mismo valle, lo que muestra un relativo grado de independencia del sector asociado a la consolidación progresiva de la centralidad suburbana secundaria de Tumbaco.

En la mañana la hora pico en cuanto a llegadas a Quito es la hora 7 - 8. Según la encuesta Origen Destino realizada en los buses urbanos e interparroquiales en noviembre de 1998 por la UPGT (Unidad de Planificación y Gestión de Transporte), el $66 \%$ de esos desplazamientos se hace con un motivo de trabajo. En la tarde la hora pico es la hora 18 - 19. El $51 \%$ de esos desplazamientos se hace para regresar al hogar.

En la salida de Quito (mismo lugar), bajan al valle 19998 personas diariamente en las 11 rutas interparroquiales. En la mañana la hora pico en cuanto a salidas de Quito es la hora 6 - 7. El $86 \%$ de esos desplazamientos se hacen con un motivo de trabajo, lo que muestra la atractividad del valle (centro de empleos secundario). En la tarde la hora pico es la hora 17 - 18. El $71 \%$ de esos desplazamientos se hacen para regresar al hogar. El valle es el destino de muchos movimientos pendulares en la tarde debido a que sigue teniendo una vocación predominante residencial con condiciones climáticas más agradables por estar a una menor altura.

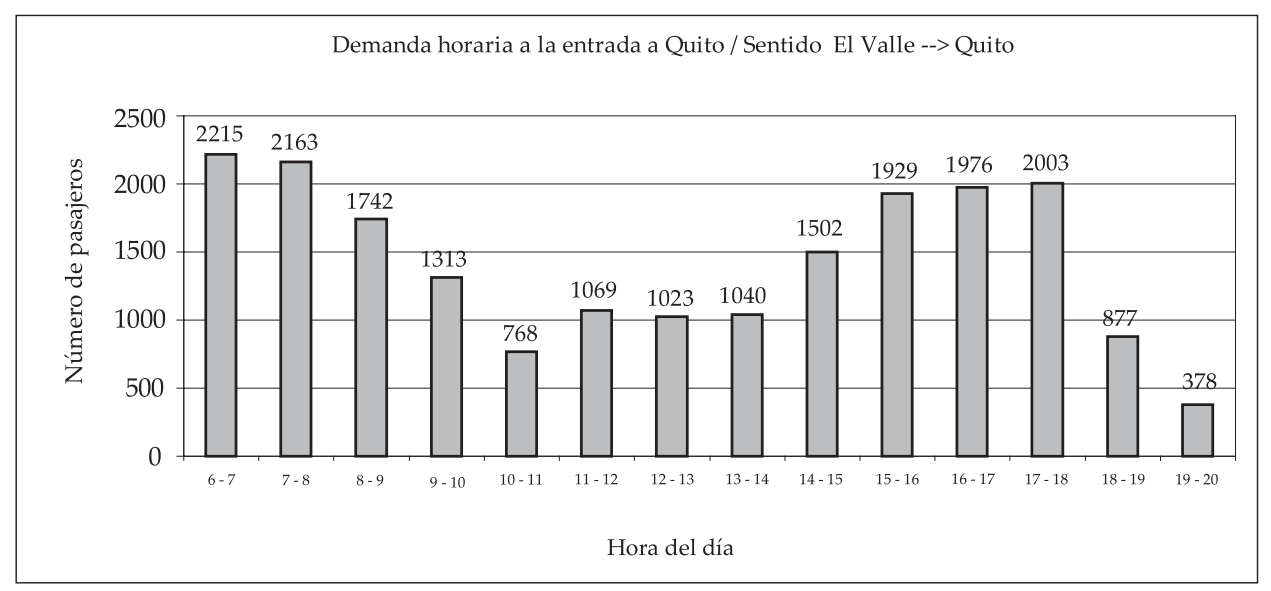

Fig. 7 - Demanda horaria a la entrada a Quito / Sentido El Valle -> Quito. 


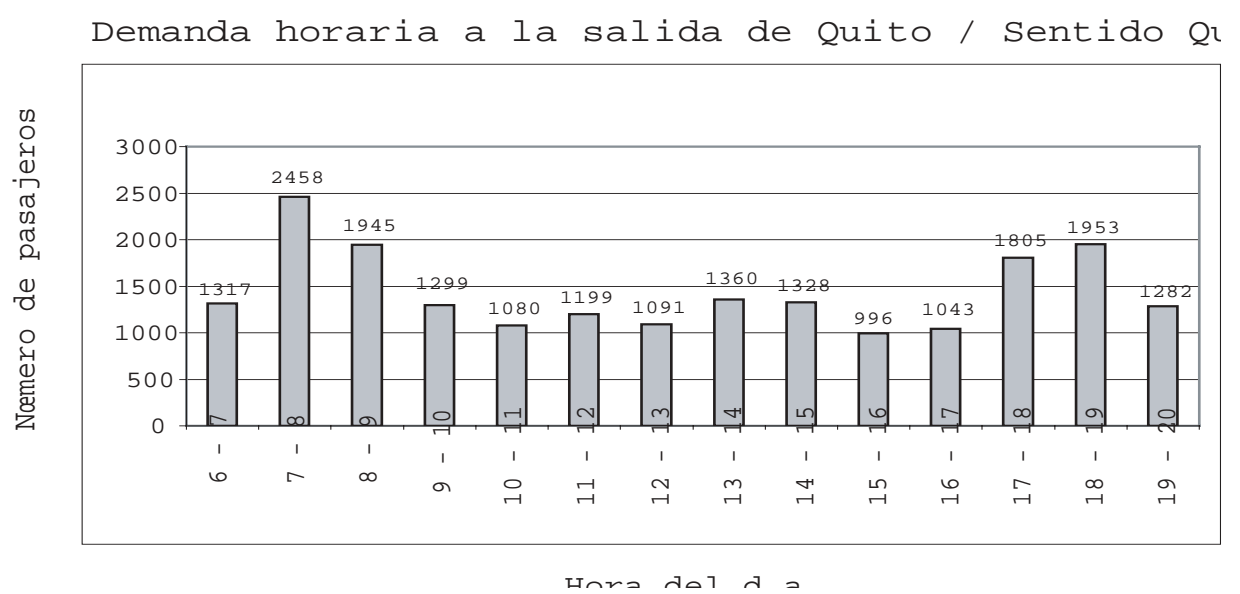

Fig. 8 - Demanda horaria a la salida de Quito / Sentido Quito -> El Valle .

\section{CONCLUSIÓN}

En el presente artículo se detalló una nueva metodología de evaluación de la demanda de transporte colectivo. Esta metodología se fomenta sobre el uso de FINDEM, un sistema innovador con GPS de recolección semiautomática de datos instalado a bordo de los buses. Este sistema permitió optimizar la realización de una encuesta Ascenso Descensos. Permitió ahorrar tiempo porque ya no fueron necesarias las anotaciones manuales en fichas de campo. Está muy adaptado al contexto de Quito ya que no existen paradas fijas; en otros términos es la única forma de conocer con precisión los sectores de mayor afluencia y de mayor llegada.

Presenta varias ventajas entre las cuales están un tecnología simple, funcional, eficaz, y desarrollada localmente. Sistemas semejantes existen en otras partes del mundo pero la importación de estos equipos es generalmente costosa y suele conllevar problemas cuando se los utiliza en países para los que no fueron diseñados.

Los datos recopilados fueron integrados a una base georeferenciada en el Sistema de Información Geográfico SAVANE. Esto permitió procesar gran cantidad de datos que fueron transformados para proponer una información sintética espacializada de gran interés para la toma de decisiones. Se elaboró también el primer plano de las rutas interparroquiales del sector nororiental de la aglomeración urbana de Quito.

Este trabajo se inserta en un proceso de evaluación general del transporte en el área metropolitana llevado a cabo por el cabildo capitalino cuyo objetivo es ajustar un modelo de transporte que permita calibrar adecuadamente la oferta y dimensionar algunas infraestructuras tales como las terminales de transferencia planificadas en el Plan Maestro de Transporte y Vialidad. Los resultados obtenidos permiten efectuar análisis relativamente precisos y detallados de demanda y condiciones operacionales del transporte colectivo. Esto puso en evidencia dinámicas hasta entonces desconocidas. Sin embargo, sin estudios adicionales tales como encuestas de movilidad en los hogares, es difícil determinar con exactitud las prácticas de movilidad. 
Por otra parte estudios semejantes permiten fiscalizar el servicio brindado por las empresas de transporte del corredor, es decir comprobar si cumplen con los permisos de operación otorgados por el ente concedente. Pueden servir además a las operadoras, para que puedan definir una estrategia operacional para mejorar el servicio y su rentabilidad. Sin embargo debido al marco artesanal de la explotación del transporte actual en Quito, son metas difícilmente alcanzables sin una reestructuración y fusiones entre operadoras lo que permitiría limitar la competencia entre ellas.

El sistema FINDEM puede ser utilizado en estudios similares de evaluación de la demanda en otras metrópolis latinoamericanas que tienen las mismas características y problemas en el ámbito de la transportación urbana. Puede además servir para el control en tiempo real o a posteriori de la operación permanente de un sistema de transporte. En especial se puede adaptar a una red integrada, tal como la del trolebús en Quito para su fiscalización una vez delegada su operación al sector privado.

Más allá de esas consideraciones técnicas, conocer en forma precisa la movilidad de los ciudadanos representa un desafío de gran envergadura para las municipalidades en el ámbito económico y político ya que el transporte es un elemento fundamental para el funcionamiento de un sistema territorial. Del manejo adecuado del transporte que requiere la disponibilidad de datos estadísticos actualizados y confiables depende el desarrollo socioeconómico de una metrópoli.

\section{Referencias citadas}

BONDOUX, F. \& DEMORAES, F., 2002 - Determinación de la demanda en transporte interparroquial e informal del corredor Quito - El Quinche mediante una encuesta Ascensos Descensos - Uso de un equipo de monitoreo satelital (FINDEM), Consultoría, $415 \mathrm{p}$.

CUSSET, J.-M., 1993 - Dynamique des systèmes de transport urbain dans les pays en développement : convergence et singularités. La Recherche-Transports-Sécurité, revue INRETS, $\mathbf{n}^{\circ}$ 37: 23-32.

DOURTHE, W. M., MALBRAN, H. \& FIGUEROA, O., 1998 - Déréglementation et reréglementation du transport public urbain : les cas de Santiago du Chili. In: La politique de déplacements urbains - Outil du développement durable : 815-819; Le Cap, 21-25 septembre 1998. Actes du congrès international CODATU VIII.

MDMQ-DMT, 2002 - Plan Maestro de Transporte y Vialidad, Síntesis, 56 p.; Quito: Municipio del Distrito Metropolitano de Quito, Dirección Metropolitana de Transporte.

SOURIS, M., 2002 - La construction d'un système d'information géographique, principes et algorithmes du système SAVANE, Thèse de Doctorat, Université de La Rochelle, 505 p. Una versión en formato pdf es disponible en la página siguiente : www.bondy.ird.fr/cvd/ pages 2003/these marc/

Adicionalmente una versión demo del SIG utilizado en el presento texto, la documentación, los manuales y unos ejemplos de utilización de Savane son disponibles en los sitios internet siguientes :

http://rslultra.star.ait.ac.th/ souris/savane/manuels.htm

http://www.bondy.ird.fr/carto/logchar/savane.html 
OTROS SITIOS INTERNET CONSULTADOS :

INRETS : Institut National de Recherche et d'Études sur les Transports : Définition d'un système embarqué d'aide à la planification et au guidage adapté aux chauffeurs routiers longue distance

http://www.inrets.fr/infos/denis/PresentationLescot/documents/Fiche\%20dantan.predit.pdf

STI : Systèmes de Transports Intelligents (Canada): Traitement de données de déplacement provenant de capteur de véhicule de transport en commun

http://www.its-sti.gc.ca/Architecture/Francais/web/TRVS.htm

\section{Siglas utilizadas}

AD : Ascensos Descensos (encuesta)

DMQ : Distrito Metropolitano de Quito

DMT : Dirección Metropolitana de Transporte y Vialidad (Municipio de Quito)

DMTV : Dirección Metropolitana de Territorio y Vivienda (Municipio de Quito)

EMSAT : Empresa Metropolitana de Servicios y Administración del Transporte (Municipio de Quito)

EMT : Empresa Metropolitana de Transporte

GPS : Global Positioning System

INEC : Instituto Nacional de Estadísticas y Censos (Ecuador)

INRETS : Institut National de Recherche et d'Études sur les Transports (Francia)

IRD : Institut de Recherche pour le Développement (Francia)

MDMQ : Municipio del Distrito Metropolitano de Quito

OD : Origen Destino (encuesta)

PMT : Plan Maestro de Transporte y Vialidad

SIG : Sistema de Información Geográfico

TC : Transporte Colectivo

UPGT : Unidad de Planificación y Gestión de Transporte (Municipio de Quito), hoy en día denominada EMSAT 